Review Article

\title{
Chinese Herbal Medicine for Aspirin Resistance: A Systematic Review of Randomized Controlled Trials
}

\author{
Ai-ju Liu, ${ }^{1}$ Hui-qin Li, ${ }^{1}$ Ji-huang Li, ${ }^{1}$ Yuan-yuan Wang, ${ }^{2}$ Dong Chen, ${ }^{2}$ \\ Yan Wang, ${ }^{2}$ and Guo-qing Zheng ${ }^{1}$ \\ ${ }^{1}$ Department of Neurology, The Second Affiliated Hospital of Wenzhou Medical University, Wenzhou 325027, China \\ ${ }^{2}$ Department of Cardiology, The Second Affiliated Hospital of Wenzhou Medical University, Wenzhou 325027, China
}

Correspondence should be addressed to Yan Wang; wywzchina@sina.com and Guo-qing Zheng; gq_zheng@sohu.com

Received 30 October 2013; Revised 15 December 2013; Accepted 16 December 2013; Published 20 February 2014

Academic Editor: Mao-Hsiung Yen

Copyright (C) 2014 Ai-ju Liu et al. This is an open access article distributed under the Creative Commons Attribution License, which permits unrestricted use, distribution, and reproduction in any medium, provided the original work is properly cited.

Aspirin resistance (AR) is a prevalent phenomenon and leads to significant clinical consequences, but the current evidence for effective interventional strategy is insufficient. The objective of this systematic review is thus to assess the efficacy and safety of Chinese herbal medicine (CHM) for AR. A systematical literature search was conducted in 6 databases until December 2012 to identify randomized controlled trials (RCTs) of CHM for AR. As a result, sixteen RCTs with a total of 1011 subjects were identified, suggesting that the interests of the medical profession and the public in the use of CHM for AR have grown considerably in the recent years. Tongxinluo capsule and Danshen-based prescriptions were the most frequently used herbal prescriptions, while danshen root, milkvetch root, Leech, and Rosewood were the most frequently used single herbs. Despite the apparent reported positive findings, it is premature to determine the efficacy and safety of CHM for the treatment of AR due to poor methodological quality and insufficient safety data. However, CHMs appeared to be well tolerated in all included studies. Thus, CHM as a promising candidate is worthy of improvement and development for further clinical AR trials. Large sample-size and well-designed rigorous RCTs are needed.

\section{Introduction}

1.1. Description of the Condition. Cerebral infarction and myocardial infarction can be fatal and seriously affect patients' living quality, and their incidence will increase greatly in recent decades, especially in developing countries, due to more work pressure and population aging $[1,2]$. Antiplatelet therapy is an important prevention measure for lowering the likelihood of myocardial infarction and stroke in high risk vascular patients [3,4]. Among antiplatelet drugs, acetylsalicylic acid, known as aspirin, became a cornerstone in the treatment of cardio/cerebrovascular diseases and has been shown to be effective in the secondary prevention of vascular events $[4,5]$. The use of aspirin can be dated back to early Hippocrates who used willow leaves, rich in Acetylsalicylic acid, to relieve the aches associated with multiple illnesses in ancient Greece [6, 7]. Reverend Edmund Stone who progressed aspirin from folk remedy to a blockbuster drug in 1763 isolated salicin, the glycoside of salicylic acid and the active ingredient of aspirin, from the bark of a willow tree in England [6, 7]. Aspirin was not born until 1897 when the Bayer company hired Felix Hoffman, a German chemist, who created acetylsalicylic acid by acetylating salicylic acid's hydroxyl ring resulting in well-tolerated compound $[6,8]$. After that time, people around the world began to appreciate its fast onset and predictable relief. However, for all that has been discovered about its beneficial effects, sometimes aspirin's effects are like an infatuation, eventually, they fade or disappear [6]. That is to say, despite the impressive efficacy and safety of low dose aspirin in preventing atherothrombosis, many patients who were receiving aspirin therapy still suffered significant clinical consequences such as stroke, myocardial infarction, and other vascular death events [9]. This clinical phenomenon was called aspirin resistance (AR). The reported frequency of AR was high and variable based on the different dosage of aspirin and biochemical method 
testing platelet function [9]. The study by Gum et al. [10] reported a 5\% prevalence of $A R$ in patients taking aspirin for prevention of atherosclerotic events, whereas another study reported a $60 \%$ rate of $\mathrm{AR}$ in patients with intermittent claudication [11]. A large systematic review including 42 studies reported a mean prevalence of AR as $25 \%$ in patients taking aspirin for secondary prevention [9]. Therefore, the intervention of $\mathrm{AR}$ is an important measure for the prevention of cardio/cerebrovascular diseases.

Aspirin resistance has been defined by clinical and/or laboratory criteria, but a generally accepted definition based on valid diagnostic criteria has not been established. The possible first description of AR has been debated since the 1980s; FitzGerald et al. [12] demonstrated that adenosine diphosphate (ADP) induced platelet aggregation was maximally inhibited by aspirin $40 \sim 80 \mathrm{mg} /$ day, but values returned to baseline with chronic administration at higher doses of up to $2,600 \mathrm{mg} /$ day. Unfortunately, "aspirin resistance" was not actually used until 10 years later when Helgason et al. [13] reported inadequate inhibition of platelet aggregation by aspirin in some patients suffering from cerebrovascular disease. However, "aspirin resistance" remains yet with no standard definition. This term used in the literature mainly included the following [14-16]. (1) Clinical "aspirin resistance" or "treatment failure" is the occurrence of acute thrombotic events despite regular aspirin therapy. (2) Laboratorydefined "aspirin resistance" or aspirin nonresponsiveness is present when in vitro platelet reactivity is not properly blocked despite the use of aspirin, ranged from the specific failure to inhibit thromboxane A2 (TXA2), failure to inhibit a test of platelet function that is dependent on TXA2 production, and the very broad failure to inhibit one or more platelet function assays. (3) "High on-treatment residual platelet reactivity" found in vitro in patients with antiplatelet therapy does not necessarily mean "resistance" to the antiplatelet drug from a pharmacological point of view. (4) Some authors distinguished types of "aspirin resistance" $[17,18]$ as follows: (a), pharmacokinetic resistance: platelet aggregability was successfully inhibited by in vivo addition of aspirin; (b), pharmacodynamic resistance: platelet aggregability continued when in vitro aspirin was added, with the persistent formation of TXA2; (c), pseudoresistance resistance: platelet aggregability was continued even when in vitro aspirin was added, but there was successful inhibition of TXA2 formation.

The key laboratory parameters are used to examine platelet responses to aspirin in a laboratory study using measures such as: (a), bleeding time: measurement of the time necessary for bleeding to stop after standardized skin incision; (b): light transmission aggregometry: analysis of light transmission after stimulation of platelets with arachidonic acid, collagen, ADP, or other agonists in anticoagulated platelet-rich plasma; (c), impedance aggregation (whole blood aggregometry): Measurement of electrical impedance between two electrodes in whole blood after stimulation with arachidonic acid, collagen, ADP, or other agonists; (d), platelet function analyzer-100 (PFA-100), assessment of aggregation under high shear; whole blood is aspirated through an aperture coated with collagen and either epinephrine or ADP; (e), rapid platelet function assay (VerifyNow Aspirin system): analysis of light transmission in a test cartridge containing fibrinogen-coated beads in whole blood; (f), levels of serum TXB2: radioimmunoassay or ELISA; TXA2 is rapidly converted into the stable metabolite TXB2; (g): urinary 11-dehydro-TXB2: measurement using radioimmunoassay or ELISA; systemic TXB2 undergoes hepatic transformation into 11-dehydro-TXB2; (h): flow cytometry: automated laser detection of platelet activation markers (e.g., P-selectin, CD63, changes in GP IIb/IIIa complex conformation) using antibodies; (i): thromboelastography: analysis of clot strength from formation to lysis after stimulation with arachidonic acid or ADP [14, 19-21].

1.2. Description of the Intervention. The current evidence for effective intervention of AR is insufficient. Clinical treatment of AR such as an increase in aspirin dose and the addition of other antiplatelet drugs is usually not effective [21, 22]. Aspirin doses of $75-150 \mathrm{mg}$ daily were at least as effective as higher doses daily, which was demonstrated in trials comparing different doses of aspirin versus no aspirin, and the addition of dipyridamole to aspirin did not reduce the incidence of ischemic events significantly comparing with aspirin alone [23]. Clopidogrel, an oral thienopyridine, can selectively and irreversible block the P2Y12 platelet receptor for ADP, thereby inhibiting ADP induced platelet aggregation. Aspirin plus clopidogrel can inhibit the two main pathways of platelet aggregation, the arachidonate/TXA2 and the ADP pathways [24]. The study by Dropinski et al. [25] reported that clopidogrel had significant effect on AR for patients with coronary artery disease. However, another study by Lev et al. [26] reported about $50 \%$ of the AR patients who were also resistant to the effects of clopidogrel because AR patients have a low inhibitory response to clopidogrel. Dipyridamole is a phosphodiesterase (PDE) inhibitor and has been used in antithrombotic treatment [27]. Although the addition of dipyridamole to aspirin has been reported be more effective in the secondary prevention of stroke than aspirin alone [28], there was insufficient evidence to indicate that dipyridamole was effective for AR in patients with vascular disease [29]. Cilostazol, an inhibitor of phosphodiesterase III, has antiplatelet aggregation activity by inhibiting the conversion of cyclic adenosine monophosphate (cAMP) to $5^{\prime}$-AMP and eventually potentiates the glycoprotein IIb-IIIa inhibitory signals to increases the level of cAMP [30, 31]. However, the current evidence only showed a tendency in supporting the additional use of cilostazol because cilostazol add-on therapy did not reduce the rate of AR [32]. Furthermore, high doses of aspirin and the addition of other antiplatelet drugs can increase the risk of gastrointestinal hemorrhage and other adverse events [22].

Faced with the limitations of the presently available treatments, complementary and/or alternative medicine (CAM) is thus increasingly being sought to treat AR worldwide. Currently, the usage of CAM has increased between the general population and medical personnel in many countries [50]. One recent study has reported that treatment of AR patients by adding one kind of CAMs, omega-3 fatty acids, 


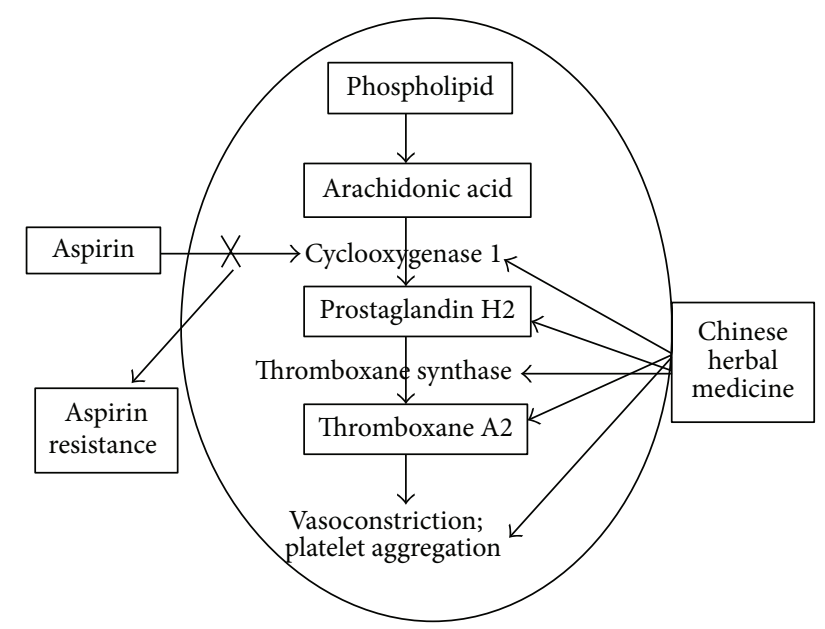

FIGURE 1: Mechanism of Chinese herbal medicine for aspirin resistance.

can improve response to aspirin and effectively reduces platelet reactivity [51]. In China, the important characteristic of China's national medical system is that traditional Chinese medicine (TCM) and Western medicine complement and cooperate with each other, being responsible together for the health care of Chinese people [52]. In the past decades, Chinese herbal medicine (CHM), as one form of CAMs, has been widely used throughout China and elsewhere in the world for the treatment of AR [53].

1.3. How the Intervention Might Work. Pharmacological studies have found the multitarget intervention effects of CHM on the pathophysiology of AR (Figure 1). For example, Buyang Huanwu decoction (BHD), a well-known classic CHM prescription for stroke, can inhibit platelet aggregation by interfering with cyclooxygenase-1 (COX-1) expression on platelet and endotheliocyte in rabbits [42]. Some CHMs for promoting blood circulation such as Rheum palmatum and Erigeron breviscapus and some tonic herbs such as Codonopsis pilosula, Astragalus membranaceus, Angelica sinensis, and ginsenosides play an important role in the inhibition of TXA2 synthesis in vitro in the porcine lung microsoma as donor of enzymes [54]. Ginkgolide C from Ginkgo biloba leaves is an inhibitor of collagen stimulated platelet aggregation by reducing TXA2 formation in rat blood sample with a dose-dependent effect [55]. The ingredients of CHM flavones can suppress platelet aggregability through inhibiting TXA2 receptor [44]. Panaxtrial saponins can inhibit platelet aggregation and TXA2 releasing induced by collagen and arachidonic acid, improve vascular endothelial function, reduce platelet surface activity, inhibit blood platelet adhesion, reduce blood viscosity, improve microcirculation, and resist thrombosis formation in patients with cardiovascular disease $[56,57]$. Atractylodis lanceae rhizoma can inhibit collagenor TXA2-induced platelet aggregation by suppressing the collagen-induced signal pathway, which is upstream of the release of TXA2 in rabbit blood sample with a concentrationdependent effect, and Poria(Indian bread) inhibits TXA2 receptor-mediated platelet aggregation by suppressing Gqmediated signaling pathway in rabbit blood sample with a concentration-dependent effect [58]. Ilexonin A, an active ingredient of Maodongqing, is a potent inhibitor of $\mathrm{vWF}$ binding and vWF mediated platelet activation and aggregation in healthy volunteer blood sample [59]. Ilexonin A can also inhibit arachidonic acid- (AA-) induced platelet aggregation by interfering with TXA2 formation in rabbit blood sample with a dose-dependent effect [60]. Danshenbased preparations have been used for hundreds of years in treatment of cardiovascular ischemic diseases [61] with the properties of inhibiting prostaglandin synthesis and platelet adhesion and aggregation in diabetic patients [62] and with suppression of both the formation and the release of TXA2 in rats [63]. Thus, CHM has the pharmacological effects of inhibiting TXA2 and consequently TXA2-dependent platelet functions.

1.4. Why It Is Important to Do This Review. Owing to the significant health risk of AR and the limitations of currently available conventional therapies, there have been a number of controlled studies over the past decade to evaluate the efficacy and safety of CHM for AR. However, to date, there was no systematic review available to determine the efficacy of CHM for AR. In addition, many of the current clinical trials of CHM for AR are thought of as "not so good" studies according to Cochrane criteria. In a TCM reviewing process, researchers may need to include such papers to identify current problems and areas worthy of improvement and future development [64]. Therefore, the objective of present systematic review is to assess the efficacy and safety of CHM for AR.

\section{Methods}

This systematic review is conducted according to the preferred reporting items for systematic reviews and metaanalyses: the PRISMA statement [65].

2.1. Eligibility Criteria. Types of studies: studies have to be randomized clinical trials (RCTs) concerning specifically the effectiveness of CHM for AR, regardless of blinding, publication status, or language. Studies of quasi-RCTs which are allocating participants by date of birth, day of the week, medical record number, and month of the year were excluded.

Types of participants: criteria for the diagnosis of AR are as follows: $>70 \%$ platelet aggregation rate induced by $10 \mu \mathrm{mol} / \mathrm{L}$ ADP and $>20 \%$ aggregation rate induced by $0.5 \mathrm{~g} / \mathrm{L}$ arachidonic acid (AA). The diagnostic criterion of aspirin semiresistance (ASR) is meeting one of the two conditions above. The exclusion criteria of AR are the following: (1) aspirin allergy or asthma; (2) all kinds of blood disease, hemorrhagic disease, or bleeding tendency; (3) platelet count $>450 \times 10^{9} / \mathrm{L}$ or $<100 \times 10^{9} / \mathrm{L}$; (4) using ticlopidine, clopidogrel, dipyridamole, unfractionated heparin, low molecular heparin, and other nonsteroidal anti-inflammatory drugs 
during the last 2 weeks of observation period; (5) active gastric ulcer patient; (6) gout patient.

Types of interventions: any intervention using $\mathrm{CHM}$ monotherapy or adjunct therapy for AR in the treatment group was included regardless of the dose, frequency, and intensity of CHM. The interventions of control groups were regular low dose aspirin treatment (aspirin $100 \mathrm{mg} / \mathrm{d}$ ), high dose aspirin treatment (aspirin $300 \mathrm{mg} / \mathrm{d}$ ), no intervention, or other antiplatelet drug treatment such as dipyridamole, clopidogrel, and cilostazol. The treatment course was at least 2 weeks in these studies.

Types of outcome measures: the primary outcome measurement was platelet aggregation rate induced by AA and/or $\mathrm{ADP}$ at the end of treatment course. The secondary outcome measurement was the clinical efficacy rate and adverse events at the end of treatment course. The clinical efficacy rate was defined as the decreased value of platelet aggregation rate before and after treatment. The standard of clinical efficacy rate met any one of the following: (1) AR becomes ASR or aspirin sensitive; (2) ASR becomes aspirin sensitive; (3) the decreased value of platelet aggregation rate is more than $10 \%$ and platelet active value is normal. The diagnosis criteria of aspirin sensitive are as follows: $\leq 70 \%$ platelet aggregation rate induced by $10 \mathrm{~mL} A D P$ and $\leq 20 \%$ aggregation rate induced by $0.5 \mathrm{~g} / \mathrm{L} \mathrm{AA}$.

2.2. Literature Search. We electronically searched CENTRAL, PubMed, EMBASE, Chinese National Knowledge Infrastructure (CNKI), VIP information database, and Wanfang Data Information Site until December 2012 to identify RCTs involving CHM for AR patients. In addition, we handsearched a list of medical journals relevant to this topic and references from relevant articles. Using free text and the medical subjects headings (MeSH) terms combined "aspirin resistance" and "Chinese herbal medicine or traditional Chinese medicine or Chinese materia medicine or integration of Chinese herbal and Western medicine."

2.3. Study Selection and Data Extraction. Two investigators independently reviewed the titles and abstracts to select potential references. Then, the two investigators read the selected articles independently and made a final decision for selection or not. Key data were extracted according to standardized criteria, including patients, methods, interventions, and outcomes. Disagreements were settled through discussion or consultation with one correspondence author.

2.4. Risk of Bias in Individual Studies. The methodological quality of RCTs was assessed independently by two investigators using the 12-item criteria from Cochrane Back Review Group [66]. All data were analyzed using Review Manager 5.0 software. We are reconciling any discrepancy through discussion with one correspondence author.

\section{Results}

3.1. Description of Studies. We identified 106 potentially relevant articles. After removal of duplicates, 103 records remained. After going through the titles and abstracts, 78 papers were excluded on the basis that they were nonclinical trials, case report, or lack of comparison group. By reading the full text of the remaining 25 articles, 7 were excluded for not being RCTs or not real RCTs; 1 paper was removed due to plagiarism; 1 paper was excluded as a result of not carrying out random method. Ultimately, 16 eligible studies were selected out for this systematic review. The screening process is summarized in a PRISMA 2009 flow diagram (Figure 2).

3.2. Characteristics of Included Studies. There were a total of 1011 subjects in the 16 included trials of CHM for AR published between 2006 and 2012. Among them, 398 participants received $\mathrm{CHM}$ adjunct therapy; 217 participants received CHM monotherapy; 396 participants received aspirin alone therapy, the addition of other antiplatelet drug to aspirin therapy, or no treatment. The age of participants ranged from 40 to 84 years old, and the treatment duration ranged from 2 weeks to 3 months. All of the studies were performed in a single center and conducted in China. These studies related to a wide range of conditions including cardiovascular disease [33, 37, 38, 40,41, 43, 46-48], cerebral infarction [34, $39,45,67]$, cardiocerebrovascular disease [35, 49], and high risk hypertension [36]. In 13 studies [33-37, 39, 41, 43, 45, $46,48,49,67]$, the diagnostic criteria of AR are an average platelet aggregation rate $\geq 70 \%$ with $10 \mu \mathrm{mol} / \mathrm{L}$ ADP as an agonist and/or $\geq 20 \%$ induced by $0.5 \mathrm{~g} / \mathrm{L}$ AA [68]. Two studies adopted a maximum platelet aggregation rate $\geq 30 \%$ induced by $0.5 \mathrm{~g} / \mathrm{L} \mathrm{AA}$ as the diagnostic criteria of AR $[38,47]$. Only 1 study [40] adopted a platelet aggregation $\geq 18 \mathrm{ohm}$ with collage or $\geq 13 \mathrm{ohm}$ with ADP as the diagnostic criteria of AR [69]. Five studies were three-group designed study [33, 37, 39-41]. In 2 studies, both CHM monotherapy and CHM adjunct therapy were compared with the regular aspirin treatment $[37,40]$. Both CHM monotherapy and CHM adjunct therapy was compared with no treatment in 1 study [33]. In two studies, CHM adjunct therapy were compared with regular aspirin treatment and the addition of other antiplatelet drug [39,41]. There was positive control in 5 studies. Among them, 3 studies used dipyridamole [34, 45, 67]; 1 study used clopidogrel [41]; 1 study used cilostazol [39]. All studies used platelet aggregation as primary outcome, and other indexes related to platelet aggregation were also used as outcome in 7 studies $[34,36,38,39,41,48,49]$. Clinical effective rate was observed in 4 studies [35, 47, 49,67], but the standard was not completely the same in each study. Adverse events were reported in 7 studies [35, 36, 38, 40, 47, 49, 67], and the incidence of adverse event in treatment group was lower than in control group. The detailed characteristics of included studies are summarized in Table 1.

3.3. Description of the CHM and Its Prescription. Seven Chinese herbal prescriptions were tested in 12 (72.5\%) of the 16 included studies, while the other 4 studies used single herb or active ingredients (Table 2). Tongxinluo capsule (TXLC) [39-41, 47] and Danshen-based prescriptions, 


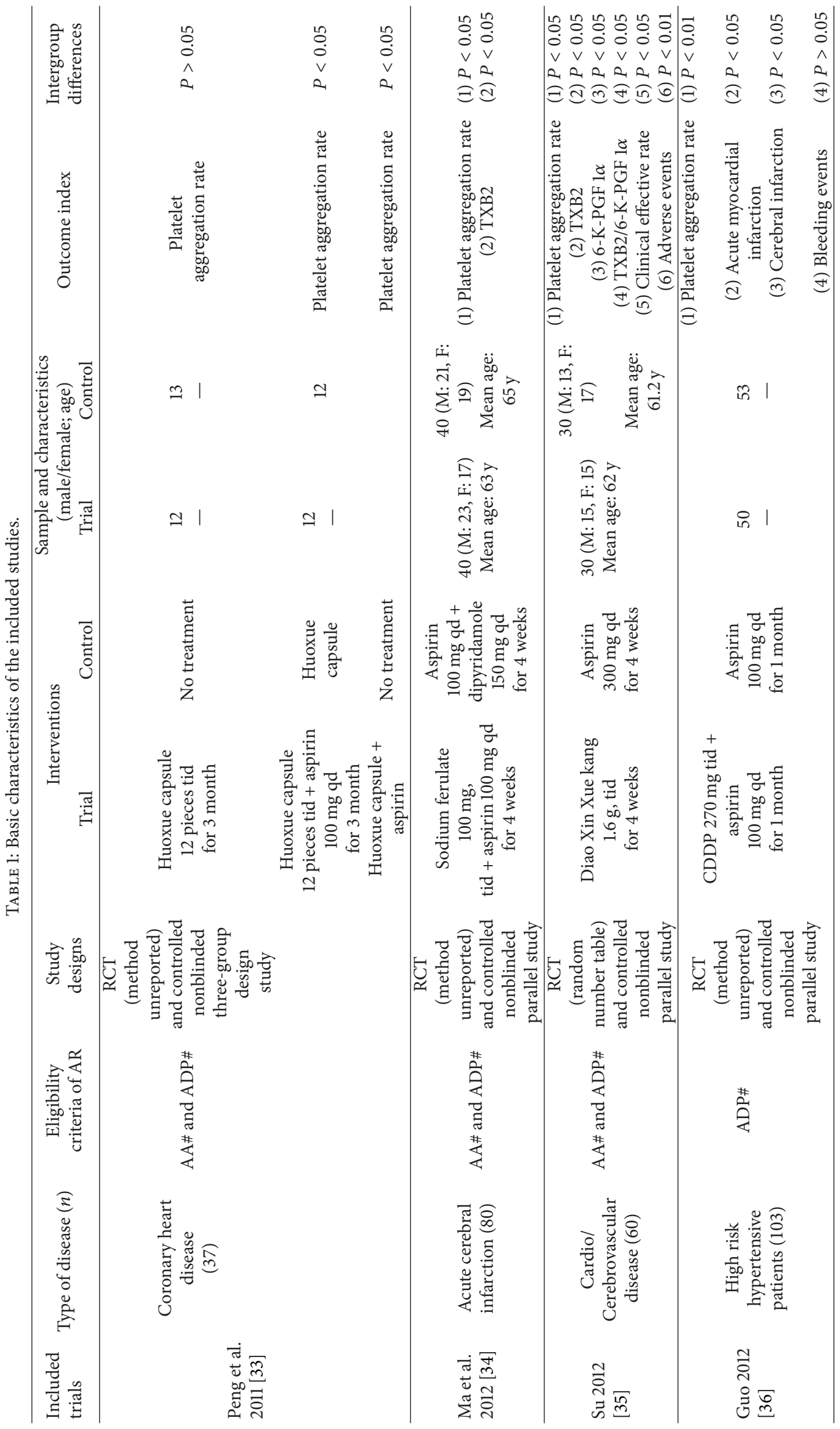




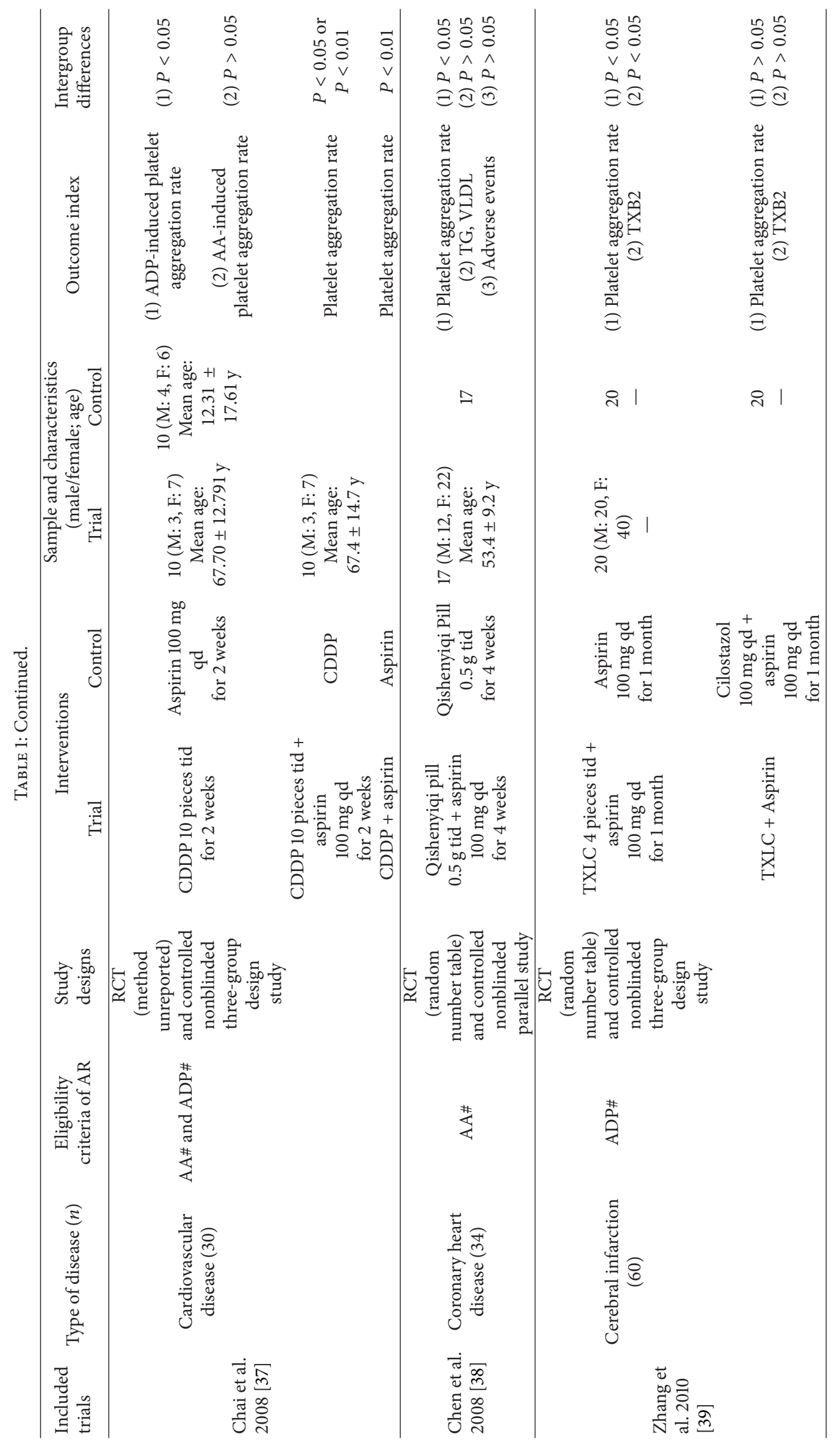




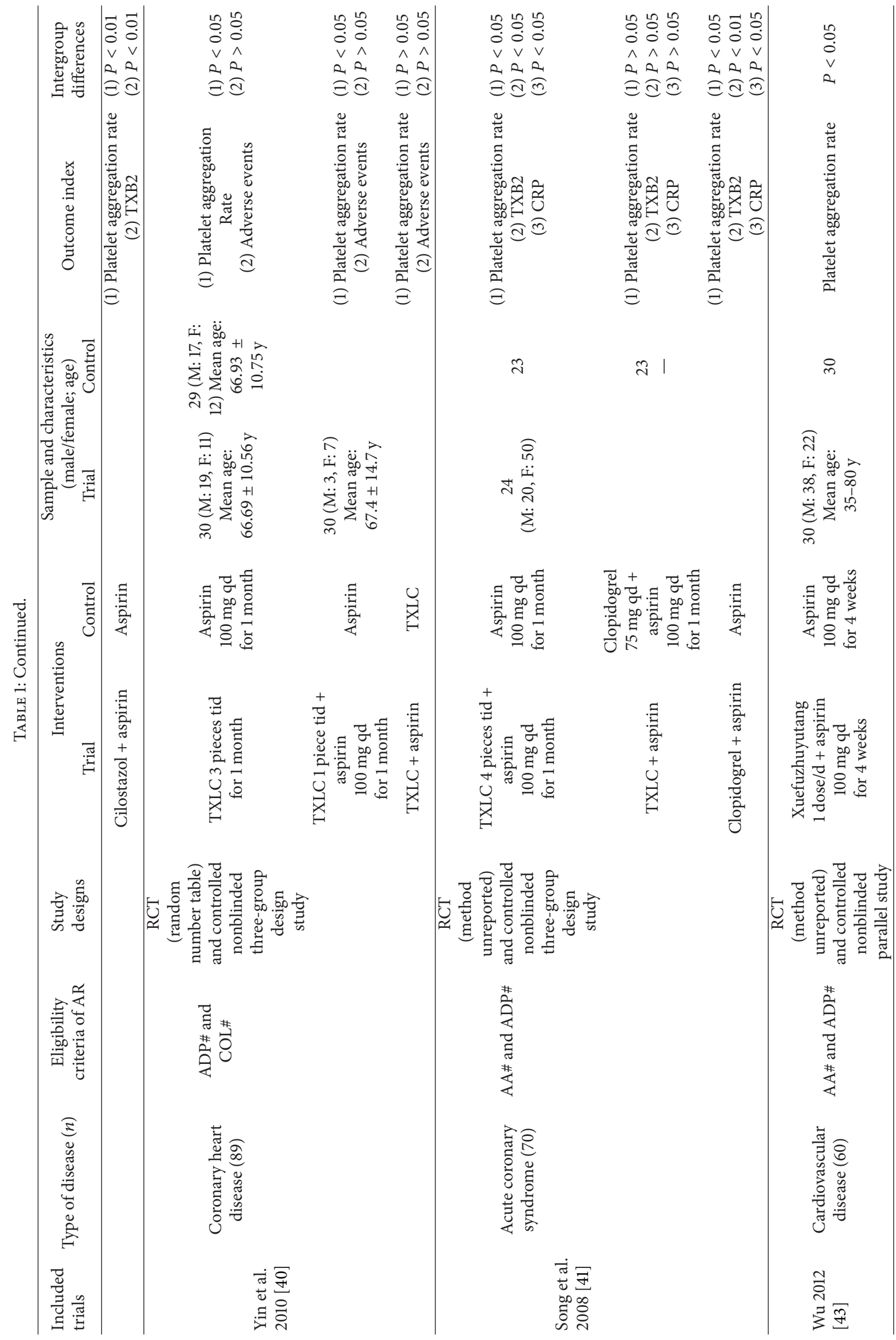




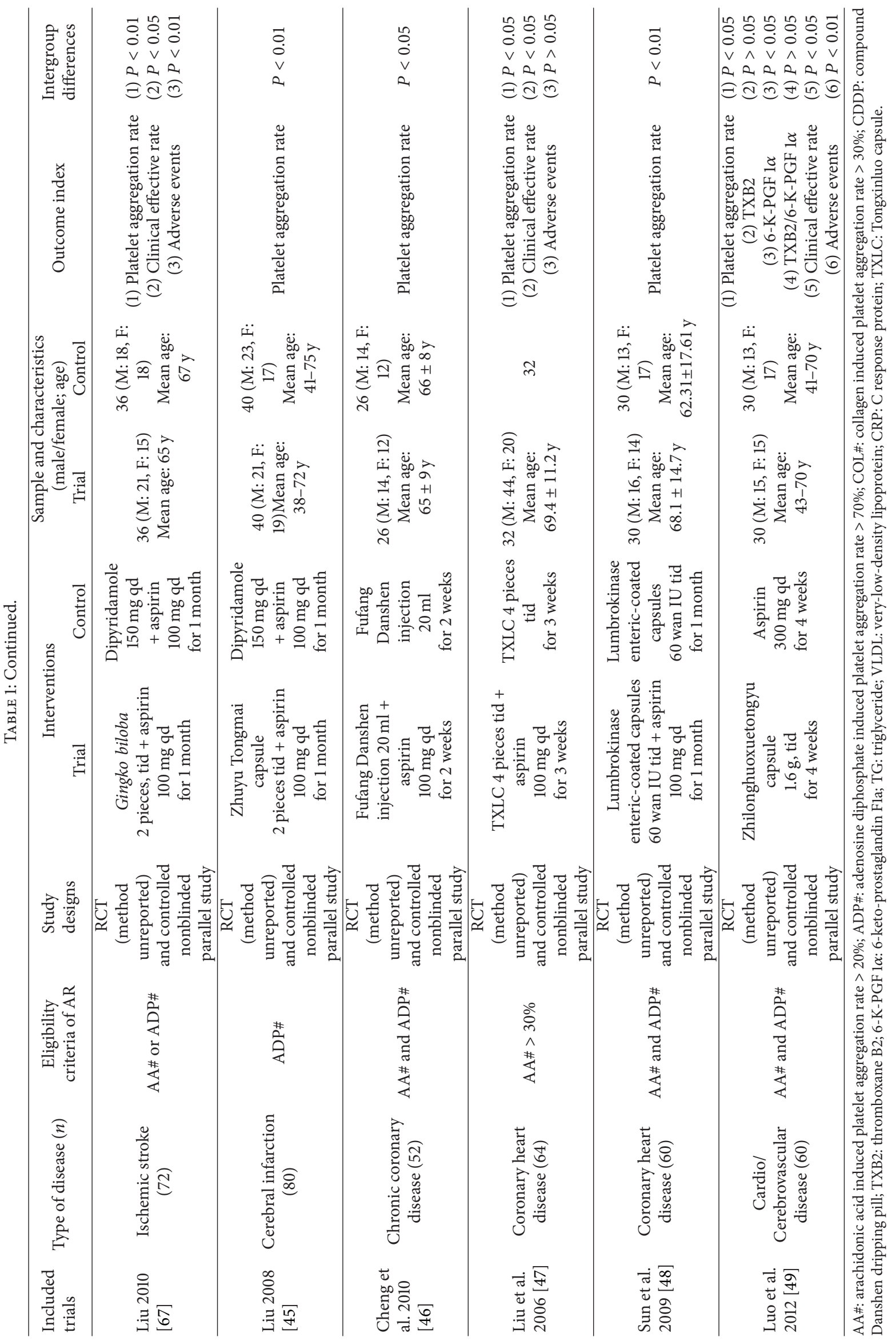


TABLE 2: Chinese herbal prescription or single herb or active ingredients for aspirin resistance in the 16 reviewed studies.

\begin{tabular}{ccc}
\hline Reference & $\begin{array}{c}\text { Chinese herbal } \\
\text { prescription or } \\
\text { single herb or } \\
\text { active ingredients }\end{array}$ & Content (Chinese pinyin, English herb name, Latin herb name, \\
Family) & Preparations/dosage & $\begin{array}{c}\text { Chinese patent } \\
\text { medicine }\end{array}$ \\
\hline
\end{tabular}

Renshen (Ginseng; Radix Ginseng; Panaxginseng C. A. Mey.), Shuizhi (Leech; Hirudo; Whitmania pigra Whitman or Hirudo nipponica Whitman or Whitmania acranulata Whitman), Quanxie (Scorpion; Scorpio; Buthus martensii Karsch), Chishao (peony root; Radix Paeoniae Rubra;

Zhang et al. 2010 [39],

Yin et al. 2010

[40],

Song et al. Tongxinluo capsule

2008 [41],

Liu et al.

2006 [47]

Paeonia lactiflora Pall. or Paeonia veitchii Lynch), Chantui

(cicada slough; Periostracum Cicadae; Cryptotympana pustulata

Fabricius), Tubiechong (ground beetle; Eupolyphaga Seu

Steleophaga; Eupolyphaga sinensis Walker or Steleophaga plancyi (Boleny)), Wusong (Centipede; Scolopendra; Scolopendra subspinipes mutilans L. Koch), Tanxiang (Sandalwood; Lignum Santali Albi; Santalum album L.), Jiangxiang (Rosewood; Lignum Dalbergiae Odoriferae; Dalbergia odorifera T. Chen), Ruxiang (Frankincense;

Olibanum; Boswellia carterii Birdw. or Boswellia bhaw-dajiana

Birdw or Boswellia neglecta M. Moore), Suanzaoren (spine date seed; Semen Ziziphi Spinosae; Ziziphus jujuba Mill. var. or spinosa (Bunge) Hu ex H. F. Chou), Bingpian (Borneol; Borneolum Syntheticum)

Chai et al. Compound 2008 [37], danshen dripping Guo 2012 [36] pill

Danshen (danshen root; Radix Salviae Miltiorrhizae; Salvia miltiorrhiza Bge)., Sanqi (Sanqi; Radix Notoginseng;

Panax notoginseng (Burk.) F. H. Chen), Bingpian (Borneol; Borneolum Syntheticum)

Danggui (Chinese angelica; Radix Angelicae Sinensis; Angelica sinensis (Oliv.) Diels) $9 \mathrm{~g}$, Shengdihuang (unprocessed

rehmannia root; Radix Rehmanniae Recens) $9 \mathrm{~g}$, Taoren (peach seed; Semen Persicae; Amygdalus persica L. or

Amygdalus davidiana (Carr.) C. de Vos ex Henry) 12 g, Honghua

(Safflower; Flos Carthami; Carthamus tinctorius L.) 9 g, Zhike (orange fruit; Fructus Aurantii; Citrus aurantium L.) $6 \mathrm{~g}$,

Chishao (peony root; Radix Paeoniae Rubra; Paeonia lactiflora

Wu 2012 [43] Xuefu Zhuyu Pall. or Paeonia veitchii Lynch) 6 g, Chuanxiong (sichuan lovage

Decoction rhizome; Rhizoma Ligustici Chuanxiong;

Dripping pill/10 pills tid

Capsule/ 3 or 4 capsules tid

Yes

Decoction//1 dose $\mathrm{qd}$

Ligusticum chuanxiong Hort.) $5 \mathrm{~g}$, Chaihu (Chinese thorowax root; Radix Bupleuri; Bupleurum chinensis DC. or Bupleurum scorzonerifolium Willd.) 3 g, Jiegeng (platycodon root; Radix Platycodonis; Platycodon grandiflorus (Jacq.) A. DC.) $5 \mathrm{~g}$, Niuxi (two-toothed achyranthes root; Radix Achyranthis Bidentatae; Achyranthes bidentata Bl.) $9 \mathrm{~g}$, Gancao

(liquorice root; Radix Glycyrrhizae; Glycyrrhiza uralensis Fisch. or Glycyrrhiza inflata Bat. or Glycyrrhiza glabra L.) $3 \mathrm{~g}$.

Xuefu Zhuyu decoction minor Jiegeng (platycodon root; Radix Platycodonis; Platycodon grandiflorus (Jacq.) A. DC.), plus Huangqi (milkvetch root; Radix Astragali seu Hedysari;

Peng et al. Huoxue capsule $2011[33]$

Astragalus membranaceus (Fisch.) Bge. var. Mongholicus (Bge.) Hsiao or Astragalus membranaceus (Fisch.) Bge.), and Suanzaoren (spine date seed; Semen Ziziphi Spinosae; Ziziphus jujuba Mill. var. or spinosa (Bunge) Hu ex H. F. Chou) Huangqi (milkvetch root; Radix Astragali seu Hedysari; Astragalus membranaceus (Fisch.) Bge. var. Mongholicus (Bge.) Hsiao or Astragalus membranaceus (Fisch.) Bge.), Danshen Chen et al. Qisheyiqi dripping 2008 [38] pill
Capsule/2 capsules tid

Dripping pill/0.5 g bid
Yes (danshen root; Radix Salviae Miltiorrhizae; Salvia miltiorrhiza

Bge.), Sanqi (Sanqi; Radix Notoginseng; Panax notoginseng

(Burk.) F. H. Chen), oil of Jiangxiang (Rosewood; Lignum Dalbergiae Odoriferae; Dallbergia odarifera T. Chen)

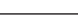


TABle 2: Continued.

\begin{tabular}{|c|c|c|c|c|}
\hline Reference & $\begin{array}{l}\text { Chinese herbal } \\
\text { prescription or } \\
\text { single herb or } \\
\text { active ingredients }\end{array}$ & $\begin{array}{l}\text { Content (Chinese pinyin, English herb name, Latin herb name, } \\
\text { Family) }\end{array}$ & Preparations/dosage & $\begin{array}{l}\text { Chinese patent } \\
\text { medicine }\end{array}$ \\
\hline Liu 2008 [45] & $\begin{array}{l}\text { Zhuoyu Tongmai } \\
\text { capsule }\end{array}$ & $\begin{array}{l}\text { Mengchong (Tabanus; Gadfly), Shuizhi (Leech; Hirudo; } \\
\text { Whitmania pigra Whitman or Hirudo nipponica Whitman or } \\
\text { Whitmania acranulata Whitman), Taoren (peach seed; Semen } \\
\text { Persicae; Amygdalus persica L. or Amygdalus davidiana (Carr.) } \\
\text { C. de Vos ex Henry), Dahuang (rhubarb root and rhizome; } \\
\text { Radix et Rhizoma Rhei; Rheum palmatum L. or } \\
\text { Rheum tanguticum Maxim. ex Balf. or Rheum officinale Baill.) }\end{array}$ & $\begin{array}{c}\text { Capsule/2 capsules } \\
\text { tid }\end{array}$ & Yes \\
\hline $\begin{array}{l}\text { Cheng et al. } \\
2010[46]\end{array}$ & $\begin{array}{l}\text { Fufang Danshen } \\
\text { injection }\end{array}$ & $\begin{array}{l}\text { Danshen (danshen root; Radix Salviae Miltiorrhizae; } \\
\text { Salvia miltiorrhiza Bge.), Jiangxiang (Rosewood; Lignum } \\
\text { Dalbergiae Odoriferae; Dalbergia odorifera T. Chen) }\end{array}$ & Injection/20 ml qd & Yes \\
\hline $\begin{array}{l}\text { Luo et al. } \\
2012[49]\end{array}$ & $\begin{array}{l}\text { Zhilong Huoxue } \\
\text { Tongyu capsule }\end{array}$ & $\begin{array}{l}\text { Huangqi (milkvetch root; Radix Astragali seu Hedysari; } \\
\text { Astragalus membranaceus (Fisch.) Bge. var. Mongolicus (Bge.) } \\
\text { Hsiao or Astragalus membranaceus (Fisch.) Bge.), Guizhi (cassia } \\
\text { twig; Ramulus Cinnamomi; Cinnamomum cassia Presl), } \\
\text { Daxueteng (sargentgloryvine stem; Caulis Sargentodoxae; } \\
\text { Sargentodoxa cuneata (Oliv.) Rehd. et Wils.), Shuizhi (Leech; } \\
\text { Hirudo; Whitmania pigra Whitman or Hirudo nipponica } \\
\text { Whitman or Whitmania acranulata Whitman), Quanxie } \\
\text { (Scorpion; Scorpio; Buthus martensii Karsch), Dilong } \\
\text { (Earthworm; Lumbricus; Pheretima aspergillum (E. Perrier) or } \\
\text { Pheretima vulgaris Chen or Pheretima guillelmi (Michaelsen) or } \\
\text { Pheretima pectinifera Michaelsen ) }\end{array}$ & Capsule/1.6 g tid & No \\
\hline $\begin{array}{l}\text { Ma et al. } 2012 \\
{[34]}\end{array}$ & $\begin{array}{l}\text { Sodium ferulate } \\
\text { tablets }\end{array}$ & $\begin{array}{l}\text { The sodium salt of ferulic acid. It is found in the root of } \\
\text { Angelica sinensis. Ferulic acid can also be extracted from the } \\
\text { root of the Chinese herb Ligusticum chuanxiong. }\end{array}$ & Tablets/100 mg tid & Yes \\
\hline Su 2012 [35] & $\begin{array}{l}\text { Diao } \\
\text { Xinxuekang } \\
\text { capsule }\end{array}$ & A dry extract of the root of Dioscorea nipponica Makino & Capsule/1.6 g tid & Yes \\
\hline Liu 2010 [67] & $\begin{array}{l}\text { Gingko biloba } \\
\text { tablets }\end{array}$ & $\begin{array}{l}\text { Extract of Yinxingye (ginkgo leaf; Folium Ginkgo; Ginkgo biloba } \\
\text { L.) }\end{array}$ & Tablets/2 tablets tid & Yes \\
\hline $\begin{array}{l}\text { Sun et al. } \\
2009[48]\end{array}$ & $\begin{array}{l}\text { Lumbrokinase } \\
\text { enteric-coated } \\
\text { capsules }\end{array}$ & $\begin{array}{l}\text { A group of proteolytic enzymes derived from the earthworm } \\
\qquad \text { Lumbricus rubellus }\end{array}$ & $\begin{array}{l}\text { Enteric-coated } \\
\text { capsule/60,000 IU } \\
\text { tid }\end{array}$ & Yes \\
\hline
\end{tabular}

including compound danshen dripping pill [36, 37], Qishenyiqi dripping pill [38], and Fufang Danshen injection [46], were the most frequently used standardized prescriptions. Danshen (danshen root; Radix Salviae Miltiorrhizae; Salvia miltiorrhiza Bge.), Huangqi (milkvetch root; Radix Astragali seu Hedysari; Astragalus membranaceus (Fisch.) Bge. var. Mongholicus (Bge.) Hsiao or Astragalus membranaceus (Fisch.) Bge.), Shuizhi (Leech; Hirudo; Whitmania pigra Whitman or Hirudo nipponica Whitman or Whitmania acranulata Whitman), and Jiangxiang (Rosewood; Lignum Dalbergiae Odoriferae; Dalbergia odorifera T. Chen) were the most frequently used single herbs, which were used for more than 3 times in all the trials. Nine of 11 preparations were Chinese patent medicine and have a rigorous quality control of herbal preparations, whereas the other 2 preparations were homemade [43, 49] (Table 2).

3.4. Risk of Bias in Included Studies. The methodological quality of RCTs was assessed independently using 12-item criteria from the Cochrane Back Review Group [66]. Risk of bias in included studies varied from $2 / 12$ to $6 / 12$, of average 3.7 . Only 4 articles $[35,38,40,67]$ reported the method of random sequences generation, although all trials claimed that they were RCTs. Only 1 trial [38] reported allocation concealment, and none of the included studies mentioned blinding. The dropout data were not reported in 5 trials [35, 38, 40, 47, 67]. All the included studies have relatively small sample sizes and do not have formal sample size calculation. The methodological quality of included studies was summarized in Table 3.

3.5. Results of Individual Studies. Meta-analysis could not be performed owing to the high clinical heterogeneity and low methodological quality of the included studies. Additionally, the number of trials is too small to draw a meaningful funnel plot. Therefore, we also did not conduct the funnel plot analysis. 


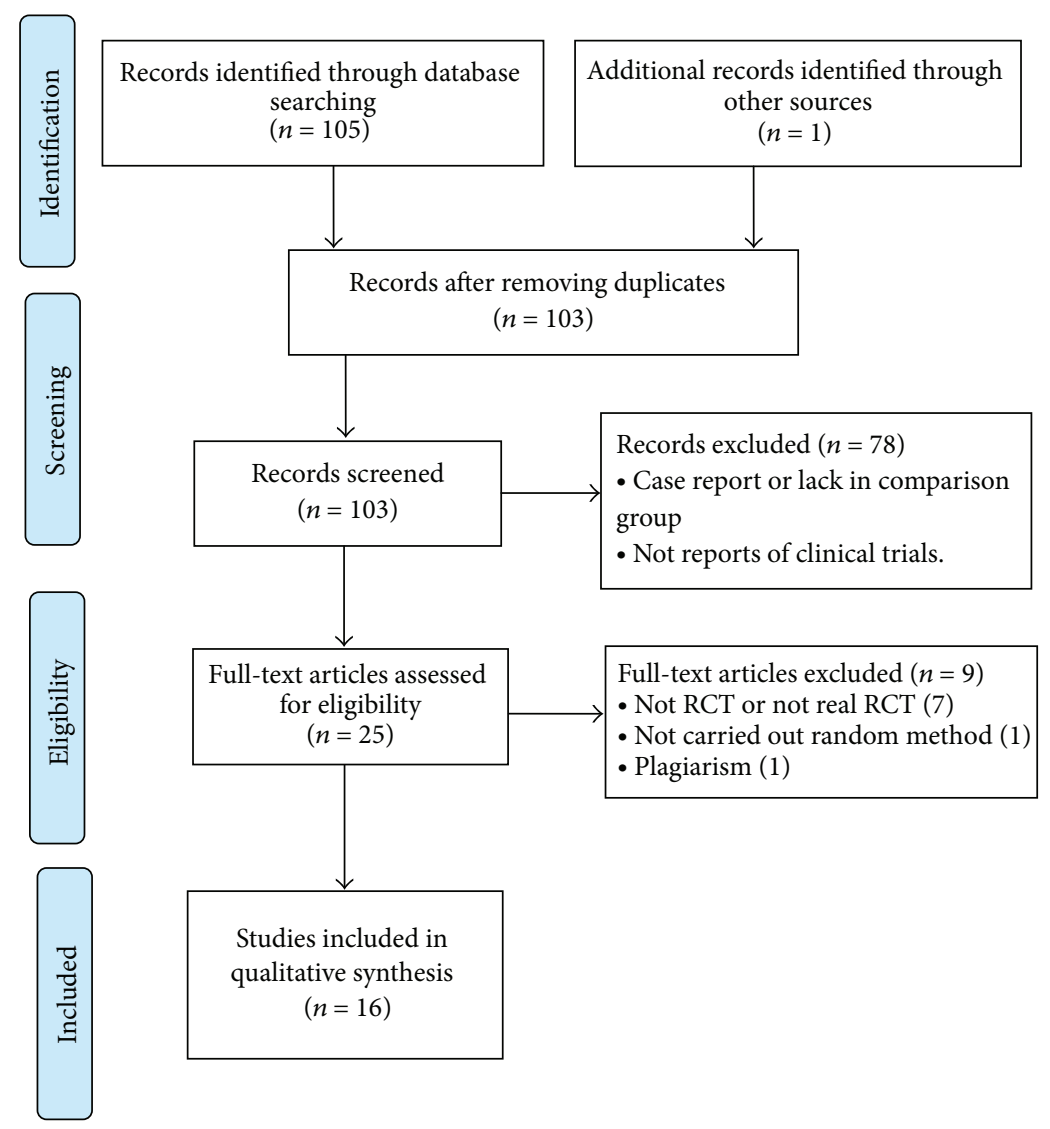

FIGURE 2: PRISMA 2009 flow diagram.

3.6. CHM Plus Aspirin versus No Treatment. One RCT [33] reported significant effects of CHM plus aspirin for reducing platelet aggregation rate compared with no treatment $(P<$ $0.05)$. Although platelet aggregation rate was partly reduced after CHM monotherapy, there was no significant difference between CHM monotherapy group and no treatment control group $(P>0.05)$, Table 1 .

3.7. CHM Monotherapy versus Aspirin $100 \mathrm{mg} / \mathrm{d}$. There are 2 RCTs comparing CHM monotherapy with aspirin $100 \mathrm{mg} / \mathrm{d}$ therapy. Yin et al. [40] reported significant effect of CHM for reducing platelet aggregation rate when compared with aspirin $100 \mathrm{mg} / \mathrm{d}(P<0.05)$. Chai et al. [37] showed that CHM has significant effect in reducing platelet aggregation induced by $\operatorname{ADP}(P<0.05)$ but has no significant effect in reducing platelet aggregation induced by AA $(P>0.05)$ (Table 1).

3.8. CHM Monotherapy versus Aspirin $300 \mathrm{mg} / \mathrm{d}$. There are 2 RCTs comparing CHM monotherapy with high dose aspirin therapy group (aspirin $300 \mathrm{mg} / \mathrm{d}$ ) [35, 49]. Studies of Luo et al. [49] and Su [35] showed significant difference in the reduction of platelet aggregation rate when CHM monotherapy was compared with high dose aspirin therapy $(P<0.05)$. Based on the testing of TXB2 and TXB2/6-K-PGF $1 \alpha$, Su [35] indicated the significant effects of $\mathrm{CHM}$ for reducing platelet aggregation $(P<0.05)$. However, Luo et al. [49] indicated that AR patients receiving Zhilong Huoxue Tongyu capsule therapy have no significant difference in comparison with high dose aspirin therapy.

3.9. CHM Plus Aspirin versus Aspirin. There were 6 RCTs comparing CHM plus aspirin with aspirin $100 \mathrm{mg} / \mathrm{d}$ therapy. All 6 trials indicated more significant effect of CHM plus aspirin for reducing platelet aggregation rate compared with aspirin $100 \mathrm{mg} / \mathrm{d}$ therapy $(P<0.05)$ [36, 37, 39-41, 43].

3.10. CHM Plus Aspirin versus Dipyridamole or Cilostazol or Clopidogrel Plus Aspirin. There were 5 RCTs that compared CHM plus aspirin therapy with dipyridamole or cilostazol or clopidogrel plus aspirin therapy $[34,39,41,45,67]$. Three RCTs [34, 45, 67] showed significant difference in reduction of platelet aggregation rate when comparing $\mathrm{CHM}$ plus aspirin therapy with dipyridamole plus aspirin therapy $(P<0.05, P<0.01)$. However, Song et al. [41] and Zhang et al. [39] indicated that AR patients receiving CHM plus aspirin therapy have no significant difference compared with cilostazol or clopidogrel plus aspirin therapy based on the rate of platelet aggregation and TX B2 production $(P>0.05)$. 
TABLE 3: The methodological quality of included studies.

\begin{tabular}{|c|c|c|c|c|c|c|c|c|c|c|c|c|c|c|c|}
\hline & $\mathrm{A}$ & $\mathrm{B}$ & $\mathrm{C}$ & $\mathrm{D}$ & $\mathrm{E}$ & $\mathrm{F}$ & G & $\mathrm{H}$ & $\mathrm{I}$ & $\mathrm{J}$ & $\mathrm{K}$ & $\mathrm{L}$ & Total + & Total - & Total? \\
\hline Peng et al. 2011 [33] & $?$ & - & - & - & - & $?$ & $?$ & $?$ & + & + & $?$ & + & 3 & 4 & 5 \\
\hline Ma et al. 2012 [34] & $?$ & - & - & - & - & $?$ & $?$ & $?$ & + & + & $?$ & + & 3 & 4 & 5 \\
\hline Su 2012 [35] & + & - & - & - & - & $?$ & + & $?$ & + & + & $?$ & + & 5 & 4 & 2 \\
\hline Guo 2012 [36] & $?$ & - & - & - & - & $?$ & - & $?$ & + & + & $?$ & + & 3 & 5 & 3 \\
\hline Chai et al. 2008 [37] & $?$ & - & - & - & - & $?$ & $?$ & $?$ & - & + & $?$ & + & 2 & 5 & 5 \\
\hline Chen et al. 2008 [38] & + & + & - & - & - & $?$ & + & $?$ & + & + & $?$ & + & 6 & 4 & 3 \\
\hline Zhang et al. 2010 [39] & $?$ & - & - & - & - & $?$ & $?$ & $?$ & + & + & $?$ & + & 3 & 4 & 6 \\
\hline Yin et al. 2010 [40] & + & - & - & - & - & $?$ & + & $?$ & + & + & + & + & 5 & 4 & 3 \\
\hline Song et al. 2008 [41] & $?$ & - & - & - & - & $?$ & $?$ & $?$ & + & + & $?$ & + & 3 & 4 & 5 \\
\hline Wu 2012 [43] & $?$ & - & - & - & - & $?$ & $?$ & $?$ & + & + & $?$ & + & 3 & 4 & 5 \\
\hline Liu 2010 [67] & + & - & - & - & - & $?$ & + & $?$ & + & + & $?$ & + & 5 & 4 & 2 \\
\hline Liu 2008 [45] & $?$ & - & - & - & - & $?$ & $?$ & $?$ & + & + & $?$ & + & 3 & 4 & 5 \\
\hline Cheng et al. 2010 [46] & $?$ & - & - & - & - & $?$ & $?$ & $?$ & + & + & $?$ & + & 3 & 4 & 5 \\
\hline Liu et al. 2006 [47] & $?$ & - & - & - & - & $?$ & + & $?$ & + & + & $?$ & + & 4 & 4 & 4 \\
\hline Sun et al. 2009 [48] & $?$ & - & - & - & - & $?$ & $?$ & $?$ & + & + & $?$ & + & 3 & 4 & 5 \\
\hline Luo et al. 2012 [49] & $?$ & - & - & - & - & $?$ & $?$ & $?$ & + & + & $?$ & + & 3 & 4 & 5 \\
\hline
\end{tabular}

A: adequate sequence generation; B: concealment of allocation; C: blinding (patient); D: blinding (investigator); E: blinding (assessor); F: incomplete outcome data addressed (ITT analysis); G: incomplete outcome data addressed (dropouts); H: free of selective reporting; I: similarity at baseline; J: cointerventions constant; K: compliance acceptable; L: similar timing outcome assessments. +: yes, -: no, and ?: unclear.

3.11. CHM Plus Aspirin versus CHM. There were 7 RCTs which compared CHM plus aspirin therapy with CHM monotherapy [33, 37, 38, 40, 46-48] (Table 1). Six RCTs demonstrated that CHM plus aspirin therapy has significant effect on reducing platelet aggregation rate when compared with CHM monotherapy $(P<0.05, P<0.01)[33,37,38,46-$ 48], whereas 1 study [40] showed no significant difference in platelet aggregation rate $(P>0.05)$.

3.12. Clinical Effective Rate. Clinical effective rate was reported in 4 out of 16 included studies [35, 47, 49, 67]. One RCT [47] indicated more significant effect of CHM plus aspirin for improving clinical effective rate compared with CHM monotherapy $(P<0.05)$. Liu et al. [67] reported significant difference in improving clinical effective rate when comparing CHM plus aspirin therapy with dipyridamole plus aspirin therapy $(P<0.05)$. Su [35] and Luo et al. [49] reported better effect of CHM for improving clinical effective rate of AR compared with high dose aspirin $(P<0.05)$.

3.13. Adverse Events. Among the 16 included studies, adverse events were reported in 7 studies $[35,36,38,40,47,49$, 67]. There were no adverse events reported in 3 studies $[38,40,47]$, while the left 4 studies reported the occurrence of adverse events, and statistical analysis was done. Guo et al. [36] reported adverse events such as acute myocardial infarction, cerebral infarction, or intracerebral hemorrhage, and the results showed that CHM was less likely to have adverse events $(P<0.05$ or $P<0.01)$. A number of mild adverse events were observed in 3 studies [35, 49,67], such as headache, dizziness, facial flushing, epigastric discomfort, and nausea, which can recover with no treatment. Liu et al. [67] reported 4 cases of adverse events in Gingko biloba plus aspirin group and 17 cases in dipyridamole plus aspirin group $(P<0.01)$. Su [35] reported 2 cases of adverse events in Diao Xinxuekang group and 16 cases in high dose aspirin group $(P<0.01)$. Luo et al. [49] reported 2 cases of adverse events in Zhilong Huowue Tongyu capsule group and 12 cases in high dose aspirin group $(P<0.01)$; see Table 1 .

\section{Discussion}

4.1. Summary of Evidence. To our knowledge, this study is the first systematic review to determine the efficacy and safety of CHM for AR patients. A total of 1011 subjects in the 16 included trials of CHM for AR have emerged between 2006 and 2012, suggesting that the interests of the public and the medical profession in the use of CHM for AR have grown considerably in the recent years. Most of herbal preparations were Chinese patent medicine, which have a rigorous quality control. The current evidence is insufficient to recommend the routine use of CHMs for AR because of the poor methodological quality and lack of safety data.

4.2. Limitations. Several methodological limitations in the primary studies should be addressed. First, although all the included trials claimed to be RCTs, only 4 trials $[35,38,40,67]$ reported the method of random sequences generation. Lack of random description would lead to the possibility of bias. Only 1 trial [38] reported allocation concealment. Inadequate or unclear allocation concealment in trials could lead to an average $18 \%$ more "beneficial" than effect estimates from trials with adequate concealment (95\% CI 5\% to 29\%) [70]. None of the included studies mentioned blinding, which may result in performance bias and detection bias. In addition, placebo control was not used in all included trials, which 
will make positive result in intervention group. Second, ITT analysis provides the least bias for a clinical trial [71]. CONSORT guidelines emphasized the necessity of ITT analysis on the reporting of randomized controlled trials [72]. However, there was no trial that mentioned analyzing data based on the ITT principle. Moreover, only 1 trial [36] mentioned 49 cases of withdrawal in the course of intervention. Third, selective bias is also a methodological limitation for this review. It is unclear if there was selective reporting in all included trials. High proportions of positive results were usually published in some Asian countries including China [73]. In this review, all included trials were from China. Therefore, we cannot exclude the possibility of selective bias. Fourth, members of the International Committee of Medical Journal Editors stated that all clinical trials must be registered in order to be publicated in September 2004 [74]. However, none of the included trials have been registered, which would affect the validity of trials. Our search strategy should have identified the majority of the available literature. However, we acknowledge the possibility that the review may not be fully comprehensive. Publication bias may also exist when nonsignificant findings remain unpublished, and thereby inflating the apparent magnitude of the effect. Fifth, an adequate sample size is vital to the design of randomized controlled trials [75]. All included studies have relatively small sample sizes and do not have formal sample size calculation, which would make this review's validity doubtable. Sixth, 12 different CHMs with great variation in terms of composition, dosage, and duration of interventions were reported in 16 included trials. There was a wide heterogeneity in the CHMs among the included studies because TCMs are composed of more than one herb and produce different therapeutic effects with different concentration proportions of the constituents [76]. This makes it difficult to recommend any specific CHM for clinical use.

\section{Concluding Remarks}

5.1. Implications for Practice. The available evidence from present systematic review is insufficient to recommend the routine use of CHM for AR because of the poor methodological quality, high clinical heterogeneity, and lack of safety data. However, CHMs appeared to be well tolerated in all included studies. Therefore, the efficacy and safety of CHM therapy for $\mathrm{AR}$ remain to be further determined. However, it should be remembered that a lack of scientific evidence does not necessarily mean that the treatment is ineffective [77]. TXLC [3941, 47] and Danshen-based prescriptions, including compound danshen dripping pill [36, 37], Qishenyiqi dripping pill [38], and Fufang Danshen injection [46], were the most frequently used standardized Chinese patent medicine. These prescriptions have potentially clinical efficiency and their preparations have a rigorous quality control, which should be the priority for clinical use of AR. In addition, Danshen (danshen root), Huangqi (milkvetch root), Shuizhi (Leech), and Jiangxiang (Rosewood) as the most frequently used single herbs may contribute in composing a fundamental prescription for AR.
5.2. Implications for Research. CHMs are widely used in the treatment of AR. There are too many unanswered questions, making it impossible to draw positive or negative conclusions. The most frequently used standardized Chinese patent medicine such as TXLC and Danshen-based prescriptions as well as the most frequently used herbs, including danshen root, milkvetch root, Leech, and Rosewood that may contribute in composing a fundamental prescription, could be promising candidates for further clinical trials of AR. Owing to serious attention to methodological quality, we recommend that the CONSORT 2010 statement [78], RCTs investigating CHM [79], and CONSORT for TCM [80], which consist of a checklist to determine study quality and rigor, respectively, should be used as a guideline for further designing and reporting of RCTs.

The safety of herbal medicines has become a major concern to both national health authorities and the general public. Thus, World Health Organization (WHO) published WHO guidelines on safety monitoring of herbal medicines in pharmacovigilance systems in 2004 [81]. However, the risks caused by drug-herb interactions, especially for those involving anticoagulant/antiplatelet drugs and CHM, are often ignored or underestimated. A recent review of potential harmful interactions between anticoagulant/antiplatelet agents and CHM indicated that the additive anticoagulant or antiplatelet effects of the CHMs, especially danshen root, Danggui (Chinese angelica; Radix Angelicae Sinensis; Angelica sinensis (Oliv.) Diels), Jiang (ginger; Rhizoma Zingiberis Recens; Zingiber officinale Rosc.), Yinxingye (ginkgo leaf; Folium Ginkgo; Ginkgo biloba L.), Gancao (liquorice root; Radix Glycyrrhizae; Glycyrrhiza uralensis Fisch. or Glycyrrhiza inflata Bat. or Glycyrrhiza glabra L.), and Jianghuang (Turmeric; Rhizoma Curcumae Longae; Curcuma longa L.), could increase bleeding risks in those patients with cardio/cerebrovascular diseases [82]. However, adverse events in the present study were reported only in 7 studies $[35,36,38$, $40,47,49,67]$. Therefore, special attention should be paid to antiplatelet drug-CHMs interactions due to the potential risks of increased bleeding. Furthermore, many reports on adverse drug reactions demonstrated that the toxic effects with the use of CHM could be associated with hepatotoxicity, nephrotoxicity, nervous system damage, and so forth [83]. Therefore, adverse events should have been reported in each included trial in order to guide the clinical use of CHM for AR. A standard reporting format for ADR that has recently been developed should be used as a guideline of reporting adverse events and ADRs in the future clinical study [84].

\section{Conclusions}

Sixteen RCTs were identified in this systematic review for evaluating the efficacy and safety of various CHMs in the treatment of AR. Despite the apparent reported positive findings, it is premature to determine the efficacy and safety of CHM for the treatment of AR due to poor methodological quality and insufficient safety data. However, CHMs appeared to be well tolerated in all included studies. Therefore, CHM as a promising candidate are worthy of improvement and development for further clinical AR trials. Large sample-size 
and well-designed rigorous RCTs are needed to accurately determine the benefits and risks of CHM for AR.

\section{Conflict of Interests}

The authors have declared that no competing interests exist.

\section{Acknowledgments}

This project was supported by the Grant of National Natural Science Foundation of China (81173395/H2902) and the young and middle-aged university discipline leaders of Zhejiang province, China (2013277).

\section{References}

[1] N. Hammar, L. Alfredsson, and T. Theorell, "Job characteristics and the incidence of myocardial infarction," International Journal of Epidemiology, vol. 23, no. 2, pp. 277-284, 1994.

[2] G. A. Donnan, M. Fisher, M. Macleod, and S. M. Davis, "Stroke," The Lancet, vol. 371, no. 9624, pp. 1612-1623, 2008.

[3] S. E. Straus, S. R. Majumdar, and F. A. McAlister, "New evidence for stroke prevention: scientific review," Journal of the American Medical Association, vol. 288, no. 11, pp. 1388-1395, 2002.

[4] G. S. F. Ling and S. M. Ling, "Preventing ischemic stroke in the older adult," Cleveland Clinic Journal of Medicine, vol. 72, supplement 3, pp. S14-S25, 2005.

[5] O. Abacı and K. O. Kılıçkesmez, "Aspirin resistance: where are we now?” Anadolu Kardiyoloji Dergisi, vol. 13, no. 4, pp. 370373, 2013

[6] J. Y. Wick, "Aspirin: a history, a love story", The Consultant Pharmacist, vol. 27, no. 5, pp. 322-329, 2012.

[7] D. B. Jack, "One hundred years of aspirin," The Lancet, vol. 350, no. 9075 , pp. 437-439, 1997.

[8] W. Sneader, "The discovery of aspirin: a reappraisal," British Medical Journal, vol. 321, no. 7276, pp. 1591-1594, 2000.

[9] M. M. C. Hovens, J. D. Snoep, J. C. J. Eikenboom, J. G. van der Bom, B. J. A. Mertens, and M. V. Huisman, "Prevalence of persistent platelet reactivity despite use of aspirin: a systematic review," American Heart Journal, vol. 153, no. 2, pp. 175-181, 2007.

[10] P. A. Gum, K. Kottke-Marchant, E. D. Poggio et al., "Profile and prevalence of aspirin resistance in patients with cardiovascular disease," American Journal of Cardiology, vol. 88, no. 3, pp. 230235, 2001.

[11] M. R. Mueller, A. Salat, P. Stangl et al., "Variable platelet response to low-dose ASA and the risk of limb deterioration in patients submitted to peripheral arterial angioplasty," Thrombosis and Haemostasis, vol. 78, no. 3, pp. 1003-1007, 1997.

[12] G. A. FitzGerald, J. A. Oates, and J. Hawiger, "Endogenous biosynthesis of prostacyclin and thromboxane and platelet function during chronic administration of aspirin in man," Journal of Clinical Investigation, vol. 71, no. 3, pp. 676-688, 1983.

[13] C. M. Helgason, K. L. Tortorice, S. R. Winkler et al., "Aspirin response and failure in cerebral infarction," Stroke, vol. 24, no. 3, pp. 345-350, 1993.

[14] N. Kuzniatsova, E. Shantsila, A. Blann, and G. Y. Lip, "A contemporary viewpoint on 'aspirin resistance,' Annals of Medicine, vol. 44, no. 8, pp. 773-783, 2012.
[15] R. Berent and H. Sinzinger, “'Aspirin-resistance'? A few critical considerations on definition, terminology, diagnosis, clinical value, natural course of atherosclerotic disease, and therapeutic consequences," Journal of Vascular Diseases, vol. 40, no. 6, pp. 429-438, 2011.

[16] S. Sanderson, J. Emery, T. Baglin, and A.-L. Kinmonth, "Narrative review: aspirin resistance and its clinical implications," Annals of Internal Medicine, vol. 142, no. 5, pp. 370-380, 2005.

[17] A.-A. Weber, B. Przytulski, A. Schanz, T. Hohlfeld, and K. Schrör, "Towards a definition of aspirin resistance: a typological approach," Platelets, vol. 13, no. 1, pp. 37-40, 2002.

[18] T. Kawasaki, Y. Ozeki, T. Igawa, and J.-I. Kambayashi, "Increased platelet sensitivity to collagen in individuals resistant to low-dose aspirin," Stroke, vol. 31, no. 3, pp. 591-595, 2000.

[19] N. Zimmermann and T. Hohlfeld, "Clinical implications of aspirin resistance," Thrombosis and Haemostasis, vol. 100, no. 3, pp. 379-390, 2008.

[20] R. FitzGerald and M. Pirmohamed, "Aspirin resistance: effect of clinical, biochemical and genetic factors," Pharmacology and Therapeutics, vol. 130, no. 2, pp. 213-225, 2011.

[21] D. Tousoulis, G. Siasos, and C. Stefanadis, "Aspirin resistance: what the cardiologist needs to know?" International Journal of Cardiology, vol. 132, no. 2, pp. 153-156, 2009.

[22] M. J. Alberts, "Platelet function testing for aspirin resistance is reasonable to do: yes!" Stroke, vol. 41, no. 10, pp. 2400-2401, 2010.

[23] C. Baigent, C. Sudlow, R. Collins, and R. Peto, "Collaborative meta-analysis of randomised trials of antiplatelet therapy for prevention of death, myocardial infarction, and stroke in high risk patients," British Medical Journal, vol. 324, no. 7329, pp. 7186, 2002.

[24] M. Cattaneo, "Aspirin and clopidogrel: efficacy, safety, and the issue of drug resistance," Arteriosclerosis, Thrombosis, and Vascular Biology, vol. 24, no. 11, pp. 1980-1987, 2004.

[25] J. Dropinski, B. Jakiela, M. Sanak et al., "The additive antiplatelet action of clopidogrel in patients with coronary artery disease treated with aspirin," Thrombosis and Haemostasis, vol. 98, no. 1, pp. 201-209, 2007.

[26] E. I. Lev, R. T. Patel, K. J. Maresh et al., "Aspirin and clopidogrel drug response in patients undergoing percutaneous coronary intervention: the role of dual drug resistance," Journal of the American College of Cardiology, vol. 47, no. 1, pp. 27-33, 2006.

[27] W. G. Eisert, "Dipyridamole in antithrombotic treatment," Advances in Cardiology, vol. 47, pp. 78-86, 2012.

[28] H. C. Diener, L. Cunha, C. Forbes, J. Sivenius, P. Smets, and A. Lowenthal, "European stroke prevention study 2. Dipyridamole and acetylsalicylic acid in the secondary prevention of stroke," Journal of the Neurological Sciences, vol. 143, no. 1-2, pp. 1-13, 1996.

[29] V. Serebruany, A. Malinin, W. Ziai et al., "Dipyridamole decreases protease-activated receptor and annexin-V binding on platelets of poststroke patients with aspirin nonresponsiveness," Cerebrovascular Diseases, vol. 21, no. 1-2, pp. 98-105, 2006.

[30] A. Hashimoto, G. Miyakoda, Y. Hirose, and T. Mori, "Activation of endothelial nitric oxide synthase by cilostazol via a cAMP/ protein kinase A- and phosphatidylinositol 3-kinase/Aktdependent mechanism," Atherosclerosis, vol. 189, no. 2, pp. 350$357,2006$.

[31] K. Nakamura, F. Ikomi, and T. Ohhashi, "Cilostazol, an inhibitor of type 3 phosphodiesterase, produces endothelium-independent vasodilation in pressurized rabbit cerebral penetrating 
arterioles," Journal of Vascular Research, vol. 43, no. 1, pp. 8694, 2006.

[32] J.-H. Lee, J.-K. Cha, S. J. Lee, S.-W. Ha, and S. U. Kwon, "Addition of cilostazol reduces biological aspirin resistance in aspirin users with ischaemic stroke: a double-blind randomized clinical trial," European Journal of Neurology, vol. 17, no. 3, pp. 434-442, 2010.

[33] N. Peng, Q. S. Liu, L. Liang, R. Li, and L. Yi, "Study on TCM syndrome distribution patterns of aspirin resistance in coronary atherosclerosis and influence of Huoxue capsule," Chinese Journal of Integrative Medicine on Cardio/Cerebrovascular Disease, vol. 9, no. 3, pp. 257-259, 2011.

[34] J. X. Ma, N. F. Gu, C. H. Xiao, C. J. Liu, and X. Y. Yin, “Clinical observation on effects of sodium ferulate on aspirin resistance in patients with cerebral infarction," Chinese Journal of Misdiagnostics, vol. 12, no. 3, pp. 530-531, 2012.

[35] W. J. Su, "DAXXK intervention clinical trials of aspirin resistance and its mechanism," China Pharmaceuticals, vol. 21, no. 15, pp. 26-28, 2012.

[36] H. Y. Guo, "Clinical studies of compound Danshen dripping pills on improving aspirin resistance in high-risk hypertension patients," Chinese Journal of Modern Drug Application, vol. 6, no. 2, pp. 74-76, 2012.

[37] Z. Q. Chai, L. Wei, and Y. Ding, "Interference of compound cardiotonic pill with aspirinresistance:a clinical trial," Practical Journal of Medicine \& Pharmacy, vol. 25, no. 4, pp. 387-389, 2008.

[38] J. Chen, D. Z. Qu, and R. Y. Wang, "Evaluation on the effectiveness of Qishenyiqi pills for aspirin resistance in patients with coronary heart disease," Chinese Traditional and Herbal Drugs, vol. 39, no. 1, pp. 96-97, 2008.

[39] J. L. Zhang, H. J. Fu, and L. B. Zhao, "Effects of tongxinluo and cilostazol on the treatment of patients with cerebral infarction," Guide of China Medicine, vol. 8, no. 21, pp. 16-18, 2010.

[40] C. H. Yin, D. P. Bi, and M. Du, "Effect of Tongxinluo capsule on platelet aggregation function in patients with aspirin resistance," Chinese Journal of Integrated Traditional and Western Medicine, vol. 30, no. 4, pp. 380-382, 2010.

[41] S. J. Song, X. Z. Zhao, Y. Wang et al., "Effects of tongxinluo capsule on C-reactive protein and thromboxan B2 in patients with acute coronary syndrome companied with aspirin resistance," Shandong Medical Journal, vol. 48, no. 31, pp. 13-15, 2008.

[42] M. Wu, Z. Q. Dong, L. M. Lou et al., "Effects of Buyang Huanwu Dection on intercellular adhesion molecule-1 and cyclooxygenase-1 expression on injured vascular endothelium in rabbits," Chinese Journal of Primary Medicine and Pharmacy, vol. 19, no. 11, pp. 1659-1660, 2012.

[43] T. H. Wu, "Study on the influence of Xuefuzhuyu Decoction on platelet aggregation in patients with aspirin resistance," Lishizhen Medicine and Materia Medica Research, vol. 23, no. 6, pp. 1586-1587, 2012.

[44] H.-B. Liu, W. Cui, J. Xu, Y. Peng, J.-J. Zhou, and P.-G. Xiao, "Molecular simulations of the inhibition of active components in traditional chinesemedicine on the thromboxane A2 receptor," Acta Physico-Chimica Sinica, vol. 26, no. 9, pp. 2549-2556, 2010.

[45] H. Q. Liu, "Clinical observation on aspirin counteract by Zhuyu Tongmai capsule," Journal of Liaoning University of Traditional Chinese Medicine, vol. 10, no. 7, pp. 109-110, 2008.

[46] G. Cheng, Y. Qiao, X. W. Yao, J. L. Yang, and X. L. Shou, "Clinical observation of the effect of compound Danshen injection on aspirin resistance," China Medicine, vol. 5, no. 9, pp. 819-820, 2010.

[47] X. C. Liu, Y. C. Hu, and M. J. Zhu, "Influence of Tongxinluo capsule on aspirin resistance in patients with coronary heart disease," Chinese Journal of Integrative Medicine on Cardio/Cerebrovascular Disease, vol. 4, no. 6, pp. 545-546, 2006.

[48] M. Y. Sun, S. J. Lv, and Y. J. Zhai, "Clinical observation of lumbrokinase enteric-coated capsule on aspirin resistance in 60 patients with coronary heart disease," Chinese Journal of Gerontology, vol. 29, no. 6, pp. 760-761, 2009.

[49] G. Luo, H. Chen, X. Bai, and S. J. Yang, "Clinical experiment intervention of Zhilong Huoxue Tongyu capsule on aspirin resistance and its mechanism," Journal of Luzhou Medical College, vol. 35, no. 1, pp. 50-52, 2012.

[50] M. Frass, R. P. Strassl, H. Friehs, M. Müllner, M. Kundi, and A. D. Kaye, "Use and acceptance of complementary and alternative medicine among the general population and medical personnel: a systematic review," Ochsner Journal, vol. 12, no. 1, pp. 45-56, 2012.

[51] E. I. Lev, A. Solodky, N. Harel et al., "Treatment of aspirinresistant patients with Omega-3 fatty acids versus aspirin dose escalation," Journal of the American College of Cardiology, vol. 55 , no. 2, pp. 114-121, 2010.

[52] Y. Wang, X.-M. Lin, and G.-Q. Zheng, "Traditional Chinese medicine for Parkinson's disease in china and beyond," Journal of Alternative and Complementary Medicine, vol. 17, no. 5, pp. 385-388, 2011.

[53] X. Y. Lu, H. Xu, and K. J. Chen, "Progress of research on aspirin resistance and its related course of integrative medical research," Chinese Journal of Integrated Traditional and Western Medicine, vol. 30, no. 6, pp. 645-648, 2010.

[54] S. R. Wang, Z. Q. Guo, and J. Z. Liao, "Experimental study on effects of 18 kinds of Chinese herbal medicine for synthesis of thromboxane A2 and PGI2," Journal of Integrated Traditional and Western Medicine, vol. 13, no. 3, pp. 167-170, 1993.

[55] H.-J. Cho, Y.-H. Shon, and K.-S. Nam, "Ginkgolide C inhibits platelet aggregation in cAMP- and cGMP-dependent manner by activating MMP-9," Biological and Pharmaceutical Bulletin, vol. 30, no. 12, pp. 2340-2344, 2007.

[56] X. M. Sun, Y. Yao, S. J. Ji, and J. B. Chen, "The effects of panax notoginseng saponin on GMP-140 and blood platelet aggregation in patients with coronary heart disease," Journal of Mathematical Medicine, vol. 14, no. 1, pp. 26-27, 2001.

[57] J. Wang, J. Xu, and J.-B. Zhong, "Effect of Radix notoginseng saponins on platelet activating molecule expression and aggregation in patients with blood hyperviscosity syndrome," Chinese Journal of Integrated Traditional and Western Medicine, vol. 24, no. 4, pp. 312-316, 2004.

[58] Y. Nasu, M. Iwashita, M. Saito, S. Fushiya, and N. Nakahata, "Inhibitory effects of Atractylodis Lanceae Rhizoma and Poria on collagenor thromboxane A2-induced aggregation in rabbit platelets," Biological and Pharmaceutical Bulletin, vol. 32, no. 5, pp. 856-860, 2009.

[59] M. Li, W.-K. Wu, L. Liu et al., "Specific inhibiting effects of Ilexonin A on von Willebrand factor-dependent platelet aggregation under high shear rate," Chinese Medical Journal, vol. 117, no. 2, pp. 241-246, 2004.

[60] T. Yang, G. F. Lin, K. D. Wang et al., "The effect of Ilexonin A administration in vivo on platelet aggregaion and malondialdehyde formation induced by Arachidonic acid in rabbit," Journal of Capital Institute Medicine, vol. 13, pp. 171-176, 1992. 
[61] T. O. Cheng, "Danshen: a versatile Chinese herbal drug for the treatment of coronary heart disease," International Journal of Cardiology, vol. 113, no. 3, pp. 437-438, 2006.

[62] X. X. Zhu, "Effect of danshan and persantin on the alteration of platelet aggregation and microcirculation in diabetics," Chinese Journal of Internal Medicine, vol. 24, no. 4, pp. 197-253, 1985.

[63] C. Z. Li, J. B. Lin, S. C. Yang, F. D. Zhao, and J. C. Zhang, "Effectof Salvia miltiorrhizae on the release of vasoconstrictive substance of platelets," Chinese Journal of Integrated Traditional and Western Medicine, vol. 4, article 565, 1984.

[64] K. Chan, D. Shaw, M. S. J. Simmonds et al., "Good practice in reviewing and publishing studies on herbal medicine, with special emphasis on traditional Chinese medicine and Chinese materia medica," Journal of Ethnopharmacology, vol. 140, no. 3, pp. 469-475, 2012.

[65] D. Moher, A. Liberati, J. Tetzlaff, and D. G. Altman, "Preferred reporting items for systematic reviews and meta-analyses: the PRISMA statement," International Journal of Surgery, vol. 8, no. 5, pp. 336-341, 2010.

[66] A. D. Furlan, V. Pennick, C. Bombardier, and M. Van Tulder, "2009 Updated method guidelines for systematic reviews in the cochrane back review group," Spine, vol. 34, no. 18, pp. 19291941, 2009.

[67] Q. K. Liu, Z. Wang, J. Xie, M. Liu, and W. H. Zhou, "Controlled clinical study on effect of Ginkgo biloba tablet on aspirin resistance," Zhejiang Journal of Traditional Chinese Medicine, vol. 45 , no. 5, pp. 320-322, 2010.

[68] P. A. Gum, K. Kottke-Marchant, P. A. Welsh, J. White, and E. J. Topol, "A prospective, blinded determination of the natural history of aspirin resistance among stable patients with cardiovascular disease," Journal of the American College of Cardiology, vol. 41, no. 6, pp. 961-965, 2003.

[69] C. H. Yin, J. C. Wang, Y. Zhao, C. M. Li, Y. T. Li, and J. H. Shao, "Correlative factors analysis of aspirin resistance in patients with coronary heart disease," Journal of Clinical Cardiology, vol. 25, no. 9, pp. 667-670, 2009.

[70] J. P. T. Higgins and S. Green, "Cochrane Handbook for Systematic Reviews of Interventions. Version 5. 0. 1, Updated March 2011," The Cochrane Collaboration, Oxford, UK, 2012, http://www.cochrane-handbook.org/.

[71] S. Oustric, S. Grill, and N. Telmon, "ITT with the penal direction," Revue du Praticien, vol. 59, supplement 8, pp. 3-7, 2009.

[72] C. Begg, M. Cho, S. Eastwood et al., "Improving the quality of reporting of randomized controlled trials: the CONSORT statement," Journal of the American Medical Association, vol. 276, no. 8, pp. 637-639, 1996.

[73] A. Vickers, N. Goyal, R. Harland, and R. Rees, "Do certain countries produce only positive results? A systematic review of controlled trials," Controlled Clinical Trials, vol. 19, no. 2, pp. 159-166, 1998.

[74] C. De Angelis, J. M. Drazen, F. A. Frizelle et al., "Clinical trial registration: a statement from the International Committee of Medical Journal Editors," The New England Journal of Medicine, vol. 351, no. 12, pp. 1250-1251, 2004.

[75] J. A. Lewis, "Statistical principles for clinical trials (ICH E9): an introductory note on an international guideline," Results Statistics in Medicine, vol. 18, no. 15, pp. 1903-1942, 1999.

[76] X. Li, H. Chen, W. Jia, and G. Xie, "A metabolomics-based strategy for the quality control of traditional chinese medicine: shengmai injection as a case study," Evidence-Based Complementary and Alternative Medicine, vol. 2013, Article ID 836179, 8 pages, 2013.
[77] V. Kotsirilos, "Complementary and alternative medicinepart 2: evidence and implications for GPs," Australian Family Physician, vol. 34, no. 8, pp. 689-691, 2005.

[78] K. F. Schulz, D. G. Altman, and D. Moher, "CONSORT 2010 statement: updated guidelines for reporting parallel group randomised trials," PLoS Medicine, vol. 7, no. 3, article e1000251, 2010.

[79] A. Flower, C. Witt, J. P. Liu, G. Ulrich-Merzenich, H. Yu, and G. Lewith, "Guidelines for randomised controlled trials investigating Chinese herbal medicine," Journal of Ethnopharmacology, vol. 140, no. 3, pp. 550-554, 2012.

[80] Z. Bian, B. Liu, D. Moher et al., "Consolidated standards of reporting trials (CONSORT) for traditional Chinese medicine: current situation and future development," Frontiers of Medicine in China, vol. 5, no. 2, pp. 171-177, 2011.

[81] WHO, WHO Guidelines on Safety Monitoring of Herbal Medicines in Pharmacovigilance Systems, World Health Organisation, Geneva, Switzerland, 2004.

[82] H. H. Tsai, H. W. Lin, Y. H. Lu, Y. L. Chen, and G. B. Mahady, "A review of potential harmful interactions between anticoagulant/antiplatelet agents and Chinese herbal medicines," PLoS One, vol. 8, no. 5, article e64255, 2013.

[83] D. Shaw, "Toxicological risks of Chinese herbs," Planta Medica, vol. 76, no. 17, pp. 2012-2018, 2010.

[84] Z.-X. Bian, H.-Y. Tian, L. Gao et al., "Improving reporting of adverse events and adverse drug reactions following injections of Chinese materia medica," Journal of Evidence-Based Medicine, vol. 3, no. 1, pp. 5-10, 2010. 


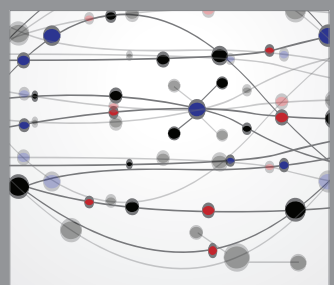

The Scientific World Journal
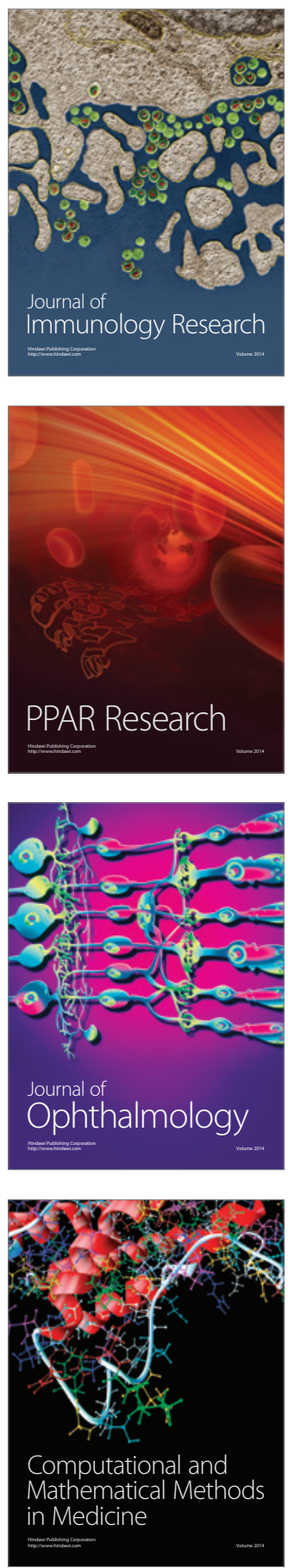

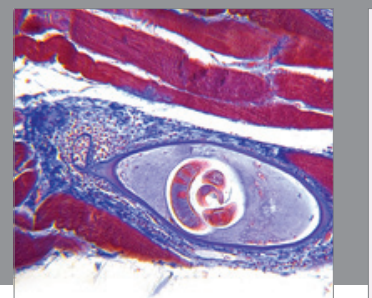

Gastroenterology

Research and Practice
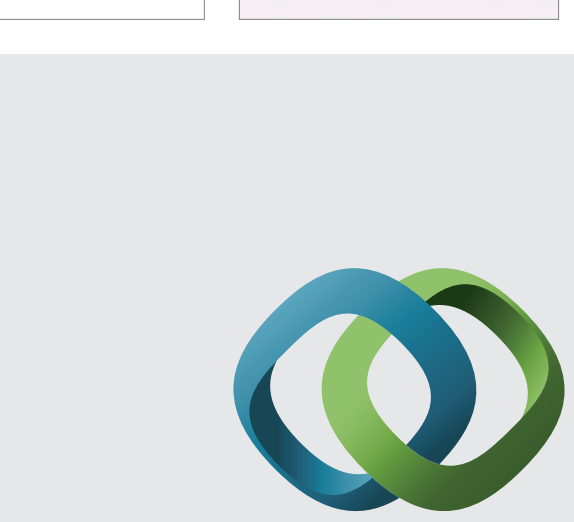

\section{Hindawi}

Submit your manuscripts at

http://www.hindawi.com
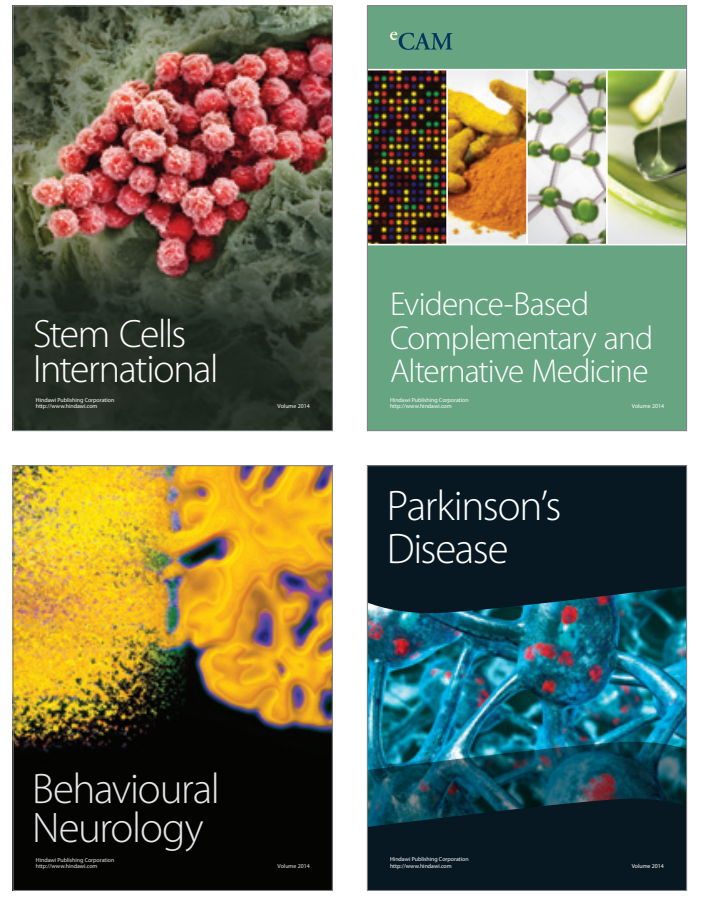
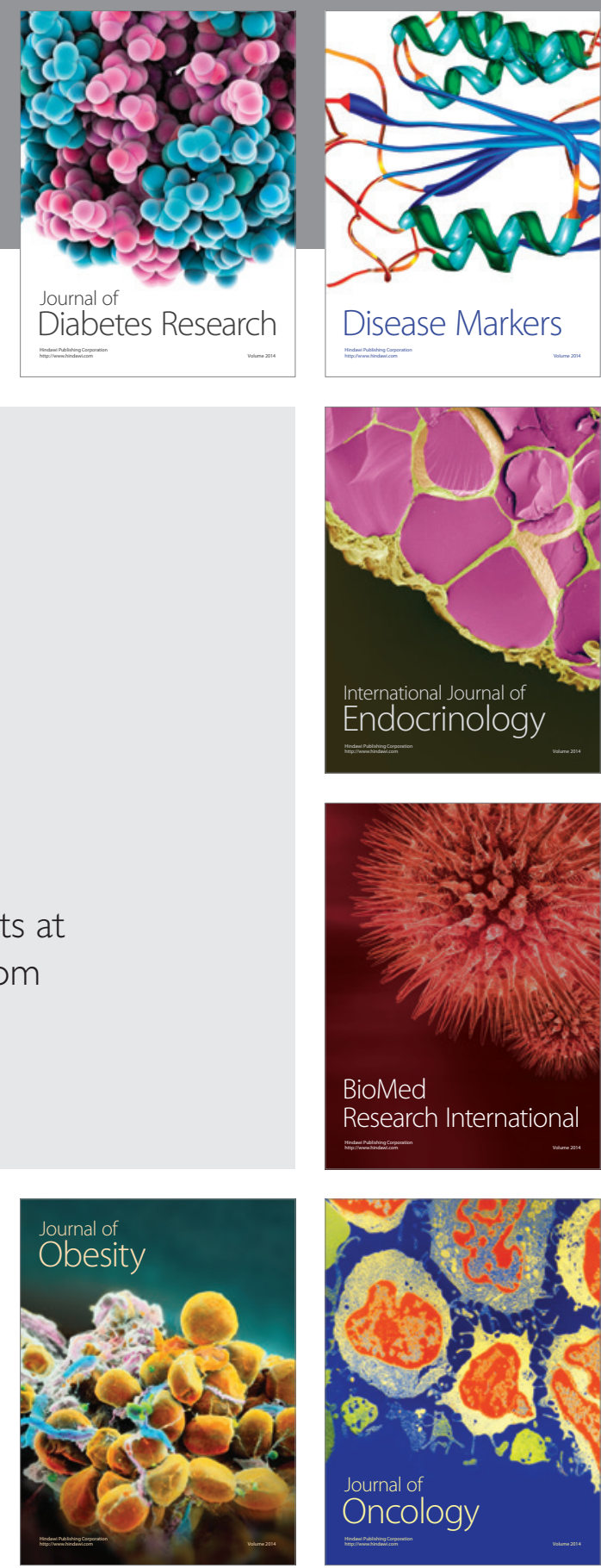

Disease Markers
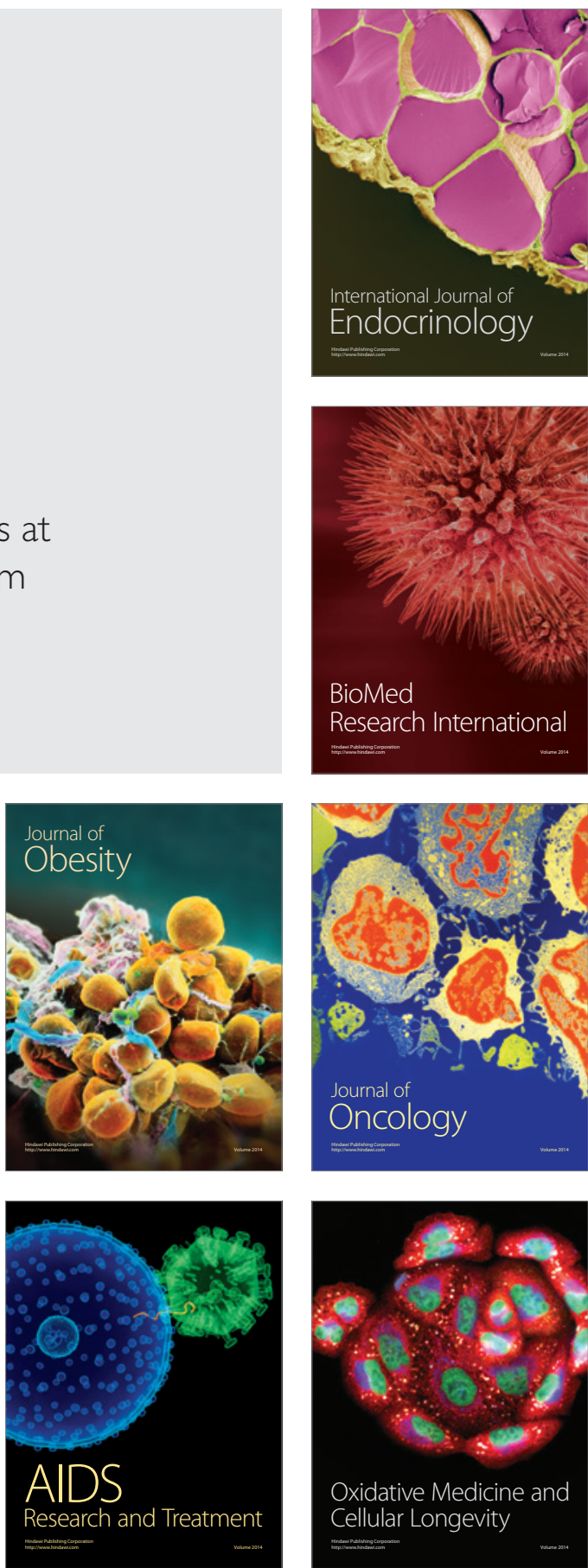